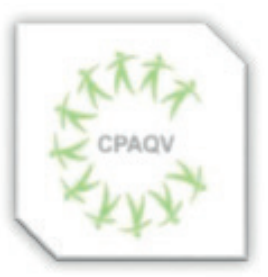

ISSN: 2178-7514

Vol. 14| No. 1| Ano 2022
ARTIGO DE REVISÃO

\section{DESEMPENHO MOTOR E FÍSICO DE ATLETAS DE FUTEBOL}

\section{Motor and physical performance of football athletes}

Adriano A. Pereira ${ }^{1}$; Uebister I. S Guedes ${ }^{7}$; José Ricardo L. Oliveira ${ }^{1}$; Ricardo P. Passos ${ }^{1,2}$; Anderson Martelli"; Anderson S. Carvalho ${ }^{1,6}$; Bráulio N. Lima ${ }^{1}$; Gustavo C. Martins ${ }^{1,9,10,11}$; Klebson S. Almeida ${ }^{1,8}$; Luis F. Silio ${ }^{1}$; Marcelo F. Rodrigues ${ }^{1}$; Mariela S. Maneschy ${ }^{8}$; Pedro P. Abdalla ${ }^{1,5}$;

Carlos H. P. Fileni' ${ }^{1}$; Guanis B. Vilela Junior ${ }^{1}$; Heleise F. R. Oliveira ${ }^{1}$; Alexandre F. Carvalho ${ }^{13}$

\title{
RESUMO
}

Dentro desse contexto, o presente artigo busca analisar o desempenho motor e físicos dos jogadores de futebol. O processo de treinamento fornecerá papel e responsabilidade a cada indivíduo, de modo que eles devem estar sempre preparados para provar a responsabilidade para ter sucesso em um modelo de treinamento específico. A prontidão dos indivíduos para aprender depende de como eles são capazes como indivíduos e são capazes de seguir orientações seguras e responsáveis. O método de revisão bibliográfico permite incluir pesquisas experimentais e não experimentais, obtendo a combinação de dados empíricos e teóricos que podem direcionar à definição de conceitos, identificação de lacunas nas áreas de estudos, revisão de teorias e análise metodológica dos estudos sobre um determinado tópico. Este método exige recursos, conhecimentos e habilidades para o seu desenvolvimento.

Palavras-chave: Desempenho; Motor; Atleta; Futebol.

\begin{abstract}
Within this context, this article seeks to analyze the motor and physical performance of soccer players. The training process will provide each individual with role and responsibility, so they must always be prepared to prove responsibility to succeed in a specific training model. The readiness of individuals to learn depends on how capable they are as individuals and are able to follow safe and responsible guidelines. The literature review method allows for the inclusion of experimental and non-experimental research, obtaining a combination of empirical and theoretical data that can lead to the definition of concepts, identification of gaps in the areas of study, review of theories and methodological analysis of studies on a given topic. This method requires resources, knowledge and skills for its development.
\end{abstract}

Keywords: Performance; Motor; Athlete; Soccer.

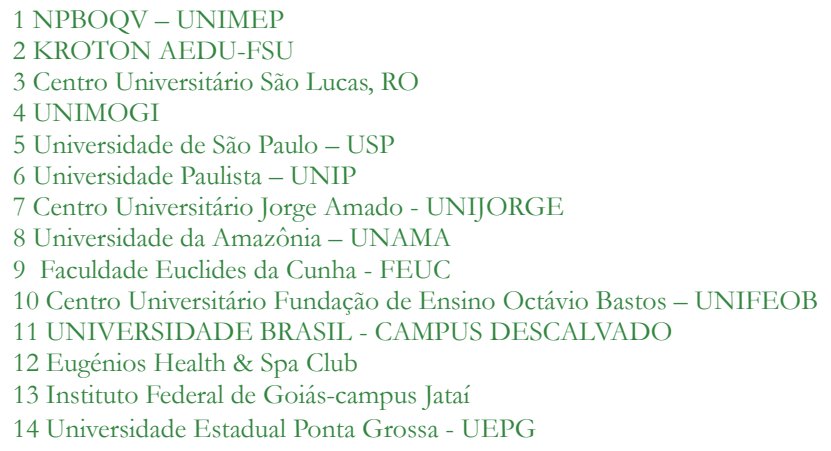

Autor de correspondência

Ricardo Pablo Passos - rppasso@gmail.com

DOI: $10.36692 / \mathrm{v} 14 \mathrm{n} 1-08 \mathrm{R}$ 


\section{INTRODUÇÃO}

O processo de aprendizagem ou treinamento vivenciado pelos atletas é conduzido continuamente, a fim de melhorar as habilidades dos atletas. Este processo é feito através da entrega de materiais que se adaptam às etapas de aprendizagem. Existem três relações sinérgicas que estão inter-relacionadas e correspondem umas às outras. Eles são chamados de domínios de aprendizagem instruídos pelos treinadores. Esses domínios de aprendizagem incluem cognitivo, psicomotor e afetivo, que devem ser abrangentes e integrados.

A relação entre os três será vivida por indivíduos com sucessos diferentes uns dos outros. Isso é muito influenciado pela habilidade de cada indivíduo. Nesse caso, a velocidade individual é muito dependente de quais domínios são mais fáceis de aceitar com base em sua respectiva prontidão. Isso influencia muito no tempo despendido no processo de treinamento e nos resultados a serem alcançados pelos indivíduos.

Da mesma forma em esportes que possuem mais orientação para as habilidades motoras, são fortemente influenciados pelo inato de cada indivíduo. Os indivíduos não são passivos, mas têm um papel ativo na implementação do modelo de instrução dado pelo instrutor em esportes, chamado de treinador ou técnico. Isso realmente depende de como o indivíduo está preparado para vivenciar o processo de treinamento ou prática.
O processo de treinamento fornecerá papel e responsabilidade a cada indivíduo, de modo que eles devem estar sempre preparados para provar a responsabilidade para ter sucesso em um modelo de treinamento específico. A prontidão dos indivíduos para aprender depende de como eles são capazes como indivíduos e são capazes de seguir orientações seguras e responsáveis.

Dentro desse contexto, o presente artigo busca analisar o desempenho motor e físicos dos jogadores de futebol.

O tipo do estudo é uma revisão bibliográfica, pesquisas do tipo tem o objetivo primordial à exposição dos atributos de determinado fenômeno ou afirmação entre suas variáveis. Assim, recomenda-se que apresente características do tipo: analisar a atmosfera como fonte direta dos dados e o pesquisador como um instrumento interruptor; não agenciar o uso de artifícios e métodos estatísticos, tendo como apreensão maior a interpretação de fenômenos e a imputação de resultados, o método deve ser o foco principal para a abordagem e não o resultado ou o fruto, a apreciação dos dados deve ser atingida de forma intuitiva e indutivamente através do pesquisador.

O método de revisão bibliográfico permite incluir pesquisas experimentais e não experimentais, obtendo a combinação de dados empíricos e teóricos que podem direcionar à definição de conceitos, identificação de 
lacunas nas áreas de estudos, revisão de teorias e análise metodológica dos estudos sobre um determinado tópico. Este método exige recursos, conhecimentos e habilidades para o seu desenvolvimento.

\section{HABILIDADES MOTORAS}

As habilidades físicas conhecidas cientificamente são as habilidades motoras, que em termos de aprendizagem motora são a base da movimentação dos atletas para a realização de todas as atividades do esporte que se pratica. Vários esportes têm necessidades específicas de habilidades motoras na forma de habilidades físicas (conhecidas como habilidades motoras). A especificidade em questão é adaptada às necessidades de movimento de cada esporte ${ }^{1}$.

Os esportes coletivos exigirão habilidades de movimento diferentes daquelas dos esportes individuais. $\mathrm{O}$ ambiente, $\mathrm{O}$ equipamento e a superfície do campo também afetarão a necessidade de tal movimento nos esportes. Ramos de esportes individuais, equipes e esportes de jogo, especialmente, precisam de fatores que determinem a necessidade de mobilidade e sucesso no treinamento de esportes, tanto internos (do jogador ou atleta) quanto externos (derivados da influência e apoio do esporte $)^{2}$.

Os fatores que determinam o sucesso podem ser na forma de: habilidades motoras, técnicas, táticas, mentais, psicomotoras, antropometria, motivação, nutrição, genética e outros fatores (apoio dos pais, amigos e espectadores e da comunidade). Habilidades motoras necessárias para apoiar a aprendizagem de habilidades como competência motora. Competência motora, como um termo global relacionado ao desenvolvimento e desempenho do movimento humano, foi definida como relacionada à proficiência em habilidades motoras fundamentais (ou seja, locomotores e habilidades de controle de objetos) ${ }^{3}$.

Assim, o termo competência de habilidade motora (MSC), conforme referido neste artigo, refere-se à competência no controle de objetos selecionados e no desempenho da habilidade locomotora. Experiências de contexto específico, incluindo prática, instrução e treinamento estruturado, são essenciais para a aquisição desses tipos de habilidades, uma vez que não se desenvolvem "naturalmente" ao longo do tempo ${ }^{4}$.

As habilidades motoras estão fortemente associadas aos fatores psicomotores e são complementares entre a necessidade de habilidade motora e o comportamento motor que cada indivíduo necessita em seus respectivos esportes. Os elementos das habilidades motoras serão capazes de correr e realizar bem as suas atividades se também forem apoiados por um bom comportamento de movimento; e vice versa ${ }^{5}$.

Destaca que para desenvolver adaptações para transferências positivas da influência do treinamento, o princípio da 
correspondência dinâmica precisa ser aplicado. O treinamento atlético não é tão simples quanto levantar peso. Existe uma relação inerente entre fatores biomecânicos, fisiológicos e psicológicos durante o exercício. Pretende-se que o papel de cada fator se apoie e se fortaleça durante o processo formativo contínuo, e até mesmo influencie positivamente o outro. A composição corporal tem um papel importante no futebol. Uma vez que no futebol ocorrem muitos contatos físicos e muitos movimentos e habilidades estão envolvidos, um alto nível de exigência física é necessário, o que envolve chutes, sprints curtos, arremessos, pegadas, armadilhas, etc. Além disso, como os jogadores de futebol precisam cobrir uma grande área do solo durante os ataques e defesas, o jogo exige preparo aeróbico e anaeróbio ${ }^{7}$.

Um grande número de acelerações e desacelerações associadas a um grande número de mudanças na direção do jogo criam uma carga adicional para os músculos envolvidos. Portanto, apenas aqueles jogadores, que são adequados para lidar com essas demandas, alcançam níveis de elite. O padrão intermitente de alta intensidade de atividade durante as partidas requer uma alta função das vias de entrega de energia aeróbia e anaeróbica ${ }^{8}$.

Além disso, a potência e a força têm grande impacto sobre o jogo que é exigido durante a corrida e na execução de várias habilidades com a bola. As habilidades biomotoras são as bases da habilidade de um indivíduo para realizar um exercício força, resistência, velocidade, coordenação, flexibilidade e agilidade. Habilidades motoras são habilidades nas atividades humanas que são influenciadas por sistemas de órgãos humanos, na forma do sistema neuromuscular (nervo), digestão, respiração, circulação sanguínea, muscular, óssea e articular? ${ }^{9}$.

Além disso, a qualidade das habilidades motoras é muito necessária para apoiar ou facilitar o desenvolvimento de processos de treinamento que serão aplicados a todos os esportes, inclusive o futebol. O termo biomotor está intimamente relacionado às habilidades motoras de que o ser humano necessita como seres vivos e em movimento. Portanto, os usos dos dois termos são muito próximos e até mesmo Intercambiáveis ${ }^{10}$.

Afirmamqueos componentesbiomotores são influenciados pela aptidão energética e pela aptidão muscular. A aptidão energética inclui capacidades aeróbicas e anaeróbicas, enquanto a aptidão muscular inclui força, resistência, velocidade, potência ou potência explosiva, flexibilidade. Habilidades de desempenho motor, em muitos anos têm sido vistas como uma construção multidimensional que consiste em componentes específicos como resistência, força, coordenação, e flexibilidade. Além disso, tornou-se óbvio que a dimensionalidade das habilidades de desempenho motor (MPA) não é a mesma para grupos-alvo diferentes como esportes de equipe. 
Neste estudo, o nível de habilidade de desempenho motor (HDM) está mais próximo do movimento de suporte do jogo como driblar, passar, controlar a bola e arremessar. A força das técnicas depende de como os jogadores empurram a bola com força e a distância dos outros jogadores para recebêla. Cada técnica precisa de alguns aspectos como força, velocidade, potência, resistência, agilidade, coordenação, equilíbrio, flexibilidade e precisão, que têm diferentes partes para aprender ${ }^{12}$.

Portanto, o HDM pode ajudar os jovens jogadores a aprender como passar para outros jogadores da maneira certa, etc. Quando os jogadores mantêm a bola e tentam chutála para o gol, eles podem chutar a bola com força ou menos força. Os jogadores podem usar a sensibilidade do toque em um dos componentes do HDM, como força suave, ao arremessar, colocando a bola longe do goleiro. Flexibilidade e equilíbrio como componentes HDM devem ser usados para manter a forma do corpo ao arremessar a bola ${ }^{1}$.

Como esta técnica de arremesso, driblar, receber e controlar e passar a bola podem ser bem feitos se os jogadores puderem aplicar os componentes HDM com precisão. Como resultado, os jogadores, principalmente os mais jovens, devem treinar o HDM e as técnicas separadamente no programa de treinamento. A fraqueza nos componentes do HDM deve ser treinada com prioridade. Outra pesquisa mostrou que 12 semanas de treinamento de velocidade, agilidade e rapidez (VAR) tiveram efeitos positivos na velocidade e agilidade dos jogadores de futebol do SAI East ${ }^{2}$.

Todos os jogadores de futebol melhoraram seu desempenho significativamente no pós-teste com o devido respeito ao pré-teste de velocidade e agilidade. O resultado revelou que o treinamento VAR é um fator importante para o aprimoramento dos jogadores de futebol. Eles também confirmam o ponto de vista de que o regime VAR é um método de treinamento importante para a melhoria da velocidade e rapidez ${ }^{3}$.

Concluíram que o treinamento VAR tem efeito positivo em jogadores de futebol nas habilidades motoras selecionadas (velocidade e agilidade). No entanto, é necessário muito planejamento para criar um programa de VAR apropriado e eficaz. Outro estudo mostrou a importância de desenvolver altos níveis de força na parte inferior do corpo para melhorar o desempenho de sprint e salto de jovens jogadores de futebol, com atletas mais fortes demonstrando desempenho superior de sprint e salto.

O futebol é caracterizado como um esporte coletivo de alta intensidade $\mathrm{e}$ contato intermitente que requer uma série de capacidades físicas e fisiológicas proficientes para ser executado com sucesso. No futebol, a força e outros aspectos do HDM apoiarão a falta de habilidades e técnicas de movimento 
individual. As habilidades de movimento em questão são ajustadas ao movimento que freqüentemente ocorre no campo e às necessidades básicas de movimento de cada esporte ${ }^{5}$.

Realizou um estudo sobre Habilidades motoras básicas: valor preditivo nos resultados de testes situacionais em jogadores de futebol de 12 a 14 anos. O objetivo principal deste estudo foi determinar o iHDMcto do sistema preditivo de variáveis (habilidade motora básica) contra o conjunto de critérios de variáveis (habilidade motora situacional). A análise dos resultados obtidos mostrou que as variáveis motoras básicas aplicadas são predominantemente responsáveis por alcançar melhores resultados nas habilidades motoras situacionais dos jogadores.

Neste estudo foram detectadas as habilidades que mais influenciam na melhora dos resultados em testes de habilidades motoras situacionais. Essas informações podem contribuir para meios mais eficientes de seleção de exercícios que seriam aplicados em programas de treinamento de jovens jogadores. Além de tudo isso, o processo de seleção de candidatos a jogadores de futebol ou atletas é conduzido para encontrar indivíduos que tenham habilidades acima da média do grupo ou do padrão de movimento que foi determinado ${ }^{7}$.

Cada parte das habilidades ou técnicas precisa de algumas fases a seguir, como fase de diversão, fase de treinamento, fase de vitória no treinamento competitivo. Portanto, o futebol pode desenvolver e explorar diferenças de estilo ou método de tempos em tempos. A capacidade de movimentação em jogos de futebol é necessária, entre outros; o movimento de caminhar, andar na ponta dos pés, correr devagar, médio e rápido, e a capacidade de agilidade, flexibilidade, equilíbrio e boa coordenação ${ }^{8}$.

Outros movimentos são na forma de movimentos de rotação simultâneos e paradas repentinas (aceleração e desaceleração). Além disso, para resistir ao iHDMcto do seu adversário (contato corporal) na tentativa de agarrar a bola (interceptar) é preciso força, velocidade e potência. Outras habilidades de movimento são como um jogador é capaz de jogar consistentemente por um longo tempo de acordo com o tempo de jogo de futebol $2 \mathrm{x}$ 45 minutos (pode ser estendido com um tempo adicional de 2 vezes 15 minutos). Portanto, pode-se concluir que se espera que os jogadores de futebol tenham boa mobilidade por 2 vezes 60 minutos 9 .

Então, para jogadores seniores, o treinamento deve ser feito com um tempo mínimo de treinamento de 2 a 3 horas. Isso é necessário como parte do princípio da prática, ou seja, a adaptação anatômica (AA) para que os jogadores se acostumem às várias situações enfrentadas em campo por 2 vezes 45 minutos ou 2 vezes 60 minutos (incluindo tempo extra 
$2 \times 15$ minutos). O futebol está muito moderno agora, tanto a partir do desenvolvimento mais completo das habilidades motoras básicas, onde se espera seja capaz de completar as técnicas e táticas em campo que acabaram se tornando as melhores habilidades suportadas por uma alta mentalidade ${ }^{10}$.

Os jogos de futebol modernos envolvem dois times se atacando com a intensidade do jogo de média a alta. A percepção no desenvolvimento de jogos de estratégia requer jogadores com jogadores da melhor qualidade em todos os elementos do jogo. Os jogadores devem ser capazes e compreender a vontade do treinador de trabalhar em campo de acordo com o cenário como estratégia para vencer uma partida. Cada movimento dos companheiros e adversários é sempre observado com atenção para que o processo de ataque e defesa corra bem $^{11}$.

Como um esporte com jogos da categoria de habilidade aberta, o futebol é jogado com o padrão de jogar um contra o outro e defender para vencer o jogo. As características do jogo de futebol têm qualificações de habilidade abertas, por isso é necessário ter a habilidade de se mover (habilidades motoras completas para lidar e superar várias situações e condições a cada mudança que ocorre no campo. O movimento dos amigos ao dominar a bola) sempre se mova e mude a qualquer momento, de modo que exija o apoio de outros coHDMnheiros de equipe para dar apoio tanto em uma área vazia da área frontal quanto na parte de trás cuja função é dar apoio a qualquer momento necessário ${ }^{12}$.

Conforme o adversário controla a bola, o movimento será sempre monitorado incluindo a possível direção da bola assim como o movimento dos adversários que sempre se movem e mudam com alta mobilidade. Este monitor requer movimento complexo e comunicação entre companheiros de equipe. Para essas necessidades, os jogadores de futebol realmente precisam do direito e boa mobilidade, todas as quais podem ser melhoradas e desenvolvidas somente por meio de treinamentos programados, sistemáticos e sustentáveis de acordo com os princípios de bons treinamentos.

\section{FUNÇÕES E} HABILIDADES COGNITIVAS EM JOGADORES DE FUTEBOL JUVENIL DE ELITE

Esportes de alta demanda requerem capacidades fisiológicas extraordinárias combinadas com habilidades excepcionais nas áreas de controle motor, percepção e funcionamento cognitivo. Duas meta-análises recentes mostraram efeitos pequenos a médios das funções cognitivas básicas em especialistas e atletas de elite que podem apontar para sua superioridade em termos de funções cognitivas basais. Além das habilidades fisiológicas, 
pesquisas anteriores focaram principalmente nas habilidades cognitivas de atletas de elite adultos ${ }^{1}$.

Em termos de atletas jovens de elite, especialmente jogadores de futebol, a pesquisa atual estudou principalmente - por um lado - os pré-requisitos físicos ou fisiológicos de jogadores de futebol juvenil de elite ou - por outro lado - os pré- requisitos psicológicos, que são as funções cognitivas de jogadores de futebol juvenil de elite em isolamento ${ }^{2}$.

Até onde sabemos, a combinação das funções motoras (ou seja, habilidades motoras específicas do futebol) e psicológicas básicas (ou seja, funções cognitivas) ainda não foi examinada. Portanto, o presente estudo é único, pois conecta aspectos psicológicos básicos (funções cognitivas) com aspectos motores (habilidades motoras específicas do futebol) de jogadores de futebol juvenil de elite ${ }^{3}$. As habilidades cognitivas referem-se à capacidade de identificar e adquirir informações ambientais de forma a integrá-las aos conhecimentos existentes. Isso permite que o indivíduo selecione e execute as respostas apropriadas. Um subgrupo especialmente interessante e importante dessas habilidades são as funções executivas (FE), que descrevem processos cognitivos que regulam pensamentos e ações, especialmente em situações não rotineiras ${ }^{4}$.

Os EF são subdivididos em EF centrais (CEF), que podem ser definidos como memória de trabalho, flexibilidade cognitiva e controle inibitório e EF de nível superior (HEF), envolvendo raciocínio, resolução de problemas e planejamento. Essas habilidades de EF amadurecem em diferentes idades, pois dependem de diferentes estruturas pré-frontais. A estrutura neuronal subjacente ao HEF é o córtex pré-frontal, que amadurece lentamente e perdura no desenvolvimento; a capacidade total é alcançada entre 20 e 29 anos de idade 5 .

Em contraste, o CEF desenvolve sua capacidade total no início da vida, na maioria das vezes antes do início da adolescência. Mais especificamente, estudos recentes mostram que (1) a cognição emerge da função motora em crianças pequenas (ou seja, 1,5-6 anos de idade) - eles previram várias funções cognitivas, como a capacidade de rotação mental, memória de trabalho, inibição e (2) que o exercício melhora a função cognitiva ${ }^{6}$.

Além disso, uma revisão de7 encontraram relações de fraco a forte entre habilidades motoras e cognitivas, especialmente em crianças pré- púberes (ou seja, com menos de 13 anos de idade), enquanto8 relatou correlações entre desempenho motor e FE em crianças com deficiência intelectual.

Afirmam que a capacidade de manter o controle motor em um ambiente esportivo imprevisível exige uma integração complexa do sistema nervoso central de entradas em constante mudança, cujo processamento também depende de funções cognitivas. No entanto, este estudo está relacionado à 
abordagem das habilidades cognitivas, visto que as funções cognitivas básicas são analisadas. Tendo em mente o valor da caça de talentos, a pesquisa atual concentra- se em jovens atletas de elite.

O presente estudo abordou a relação entre funcionamento cognitivo e habilidades motoras específicas em jogadores de futebol juvenil de elite. $O$ objetivo era ampliar o conhecimento da relação entre as habilidades cognitivas básicas e as habilidades motoras específicas do futebol. Os resultados mostraram que a diagonal AW foi positivamente correlacionada com o desempenho no drible. Isso pode sugerir que os atletas com AW mais ampla também possuem habilidades avançadas de drible.

Além disso, esses achados podem implicar que um amplo AW melhora as habilidades dos jogadores em relação a tarefas motoras altamente exigentes, pois eles podem ser capazes de perceber muitos estímulos ópticos em seu AW visual. Isso pode permitir que eles executem reações precoces em seu sistema sensório-motor para tornar seu desempenho mais eficiente. Por exemplo, numa situação de jogo onde o atleta está driblando e ao mesmo tempo tem que ficar de olho na bola, nos coHDMnheiros e nos adversários.

Neste caso, um amplo AW poderia ser benéfico, por exemplo, para evitar o contato com adversários e dribles em espaços já cobertos por coHDMnheiros de equipe. Esses resultados estão de acordo com a meta-análise anterior que implicou habilidades cognitivas superiores em atletas de elite. Outra relação positiva foi relatada para WMC e dribles, bem como para controle de bola, malabarismo com bola e pontuação total. Especialmente essas descobertas relacionadas ao WMC estão de acordo com os estudos que examinam as funções cognitivas em atletas de elite mencionados anteriormente.

Pesquisas anteriores, por exemplo, indicaram (a) que uma maior capacidade de MO está associada a um desempenho atlético superior, bem como (b) que o tempo gasto em esportes organizados se correlaciona positivamente com a MO . No entanto, existem também outros estudos que não indicaram essa relação.

Além disso, a falta de correlação dos testes motores com o teste MOT e PL pode ser devido ao fato de que as tarefas motoras não incluem demandas semelhantes. Por exemplo, nos testes de desempenho motor usados aqui, não há tarefa em que vários objetos ou jogadores precisem ser rastreados simultaneamente. Portanto, não há situação que exija habilidades semelhantes ou tenha a mesma estrutura de tarefas do MOT. Embora vários estudos relacionados à abordagem de acoplamento percepção-ação. Já provou a ligação de habilidades perceptivas e desempenho específicas, este é o primeiro estudo que sabemos que mostra uma correlação positiva 
entre as pontuações acumuladas de todos os testes cognitivos básicos e todos os testes motores que podem apontar para uma forte interação de habilidades físicas e psicológicas.

Esses resultados estão alinhados com a influência mútua das redes cognitivas e motoras em um nível funcional básico e estrutural. Além disso, eles também podem ser uma dica para o uso de redes neurais semelhantes e das mesmas regiões do cérebro ao realizar diferentes tarefas cognitivas e habilidades motoras ${ }^{10}$.

Além disso, essas descobertas estão de acordo $\operatorname{com}^{11}$ que afirmam que a capacidade de manter o controle motor em um ambiente esportivo imprevisível exige uma integração complexa do sistema nervoso central de um perfil em constante mudança de entradas sensoriais. Além disso, também está em consonância com sua afirmação de que a incorporação de elementos cognitivos que variam de tarefas duplas, resposta a estímulos, antecipação, tomada de decisão e movimento de programação em relação a alvos externos pode degradar o controle neuromuscular em relação ao movimento sem tais fatores.

Em termos de interações do desenvolvimento motor-cognição, esses resultados apontam na mesma direção que a pesquisa anterior fez com (1) crianças mais novas, (2) melhorias cognitivas em função do exercício físico, e (3) fortes relações motorcognições em crianças pré-púberes (ou seja, com menos de 13 anos de idade) 12 .
Embora os resultados sejam baseados em um estudo transversal e aguardem replicação em um desenho que permita interpretações causais, os dados revelam uma possível explicação para as diferenças de desempenho entre jogadores de futebol juvenil de elite em termos de função cognitiva e habilidades motoras específicas. WMC e AW podem ser relevantes para fins de identificação de talentos, visto que estão fortemente associados a malabarismo com bola, drible com bola e, especialmente, a pontuação total de habilidades motoras e ritmo, que são todos de grande interesse em clubes de futebol profissional ${ }^{1}$.

Ao adicionar esses testes cognitivos aos físicos (aqueles que se correlacionaram significativamente, ou seja, drible, controle de bola, malabarismo de bola e a pontuação total), as impressões e os valores derivados dos testes físicos poderiam ser fortalecidos e, além disso, as informações sobre os perfis dos jogadores em termos de função cognitiva seriam estendidas ${ }^{2}$.

Em termos de desenvolvimento de talentos, jogar futebol com alto nível de desempenho a cada dia, ou seja, em uma equipe de seleção de talentos de um clube profissional de futebol, parece estar associado ao desenvolvimento da maioria das habilidades cognitivas. Isso pode indicar que essas habilidades cognitivas podem ser cruciais para o desenvolvimento de talentos e podem ser promovidas por meio desses programas de 
talentos de clubes de futebol profissional - um desenvolvimento recíproco positivo ${ }^{3}$.

No entanto, não podemos tirar conclusões causais com base em nossos dados, pois o desenvolvimento de talentos pode ser influenciado por uma terceira variável. No entanto, até onde sabemos, este estudo é o primeiro que examina a combinação de várias funções cognitivas e habilidades motoras específicas do futebol em jovens especialistas em futebol ${ }^{4}$.

O teste motor usado analisa habilidades motoras específicas do futebol básicas, como driblar e fazer malabarismos, que são capazes de distinguir jogadores de futebol de elite de jogadores recreativos, mas não de elite de subelite. O estudo, portanto, não cobre todo o espectro do complexo jogo de futebol. Um exemplo é que nenhum HEFs foi avaliado, o que também é crucial para o jogo complexo. Portanto, algumas habilidades táticas essenciais, como MOT em um ambiente dinâmico, como jogos de pequenos lados, estão faltando5.

Além disso, embora o teste de mudança de direção seja bem validado, é uma limitação, pois carece de estímulos externos dos quais as mudanças de direção dependem em uma situação de jogo real. A diferenciação entre mudança de direção e agilidade é crucial nesse sentido. Não há situação no jogo em que um jogador tenha que mudar sua direção em uma ordem predefinida. Além disso, um grande número de correlações em nosso estudo não foi significativo. Assim, mais pesquisas de replicação neste campo são claramente necessárias. Além disso, o estudo carece de grande poder estatístico, pois a amostra única é relativamente pequena devido ao fato de que jogadores de futebol juvenil de elite foram examinados, cuja acessibilidade é estritamente limitada na maior parte do tempo6.

Consequentemente, existem várias recomendações para pesquisas futuras derivadas das limitações deste estudo. Primeiro, vinculando resultados de testes cognitivos (especialmente HEF) a (1), que são capazes de medir habilidades mais complexas e diversificadas de futebol e esportes específicos. Isso é necessário para expandir o conhecimento sobre essas correlações (por exemplo, jogos pequenos e teste de agilidade com estímulos externos) e (2), medidas de desempenho objetivas (por exemplo, avaliação de desempenho durante o jogo), e para fortalecer possíveis relações e replicar descobertas de estudos anteriores ${ }^{7}$.

No entanto, deve-se notar que os objetivos de desempenho, como gols marcados, são difíceis de medir em jovens atletas devido à grande variação nas posições dos jogadores e ao fato de que a probabilidade de marcar gols varia muito dependendo da posição. Em relação ao (1), será um desafio incluir esses testes, pois podem ocorrer problemas em termos de objetividade e confiabilidade ${ }^{8}$. 
Emsegundolugar,medidaslongitudinais com um número maior de jogadores são necessárias para examinar a interação relacionada à idade das funções cognitivas, habilidades motoras específicas do futebol e seu desenvolvimento. Especialmente considerando o tempo individual de maturação das funções cognitivas. Além disso, o uso de desenhos de estudos longitudinais permitiria aos pesquisadores pesquisar fatores influentes adicionais, bem como concluir e descobrir outras relações causais, uma vez que essa possibilidade é muito limitada em estudos transversais. Por fim, as investigações dos CEHs são necessárias, pois também são um aspecto importante do jogo complexo?.

Para resumir, descobrimos que as funções cognitivas AW e memória de trabalho estão parcialmente associadas a algumas habilidades motoras específicas e essenciais, enquanto a soma de todas as habilidades cognitivas e motoras também estão fortemente correlacionadas. Além disso, o teste cognitivo, teste MOT e PL, não apresentou relação com as habilidades motoras testadas. Embora seja preciso ter em mente que esta é apenas uma primeira tentativa de compreender a relação entre o comportamento cognitivo e motor, podemos dar uma olhada na direção para a qual esses resultados podem apontar ${ }^{10}$.

Ou seja, essas descobertas podem ser importantes tanto do ponto de vista teórico quanto prático. Do ponto de vista teórico, isso pode destacar a importância dos modelos de treinamento cognitivo que são baseados no conhecimento neurocognitivo, bem como a necessidade de modelos e teorias mais sofisticadas que explicam a relação dos movimentos, isto é, técnica e cognição. O uso de tais modelos de treinamento aumenta as funções cognitivas e possivelmente as habilidades técnicas motoras, o que está em linha com os resultados acima mostrando correlações de ambas as habilidades. $1^{1}$

A necessidade emergente desses modelos de treinamento cognitivo está em consonância com a crescente evidência de que algumas dessas funções cognitivas como AW e MOT, bem como a memória de trabalho, são treináveis. No entanto, a transferência para o jogo real ainda não está claramente estabelecida para todas as melhorias. Além disso, pesquisas sugerem que uma combinação de treinamento cognitivo e físico é mais benéfica para os atletas em termos de funções cognitivas, saúde mental e neurogênese do que apenas uma delas conduzida separadamente ${ }^{12}$.

\section{CONSIDERAÇÕES FINAIS}

Do ponto de vista prático, o conhecimento sobre a relação entre cognição e habilidades motoras, ou seja, entre a interação cérebro-músculo, poderia ajudar os clubes esportivos a buscarem talentos e novos jogadores de forma mais eficaz e holística. 
Este sofisticado sistema de aferição pode ser criado adicionando-se uma ferramenta cognitiva de aferição ou teste às categorias de técnica, atletismo e tática. A adição desta ferramenta cognitiva é apoiada por estudos que relatam uma alta ligação e sobreposição entre as funções cognitivas e a inteligência do jogo, o que é crucial para o sucesso em esportes de elite, e ainda dificilmente mensurável.

Além disso, esse conhecimento pode ser usado por treinadores para aprimorar as habilidades cognitivas de seus jogadores e, eventualmente, algumas de suas habilidades motoras, bem como melhorar a memória de trabalho para habilidades motoras gerais. Referindo-se a isso, programas individuais de treinamento de futebol poderiam ser criados com base nessas relações para melhorar o desempenho do futebol em campo.

Como esses resultados podem apontar na mesma direção que a abordagem de acoplamento percepção-ação, talvez possa ajudar os treinadores a criar programas de treinamento e exercícios que não isolem a percepção específica do esporte (ou seja, funções cognitivas) e ação (ou seja, habilidades motoras), mas em vez disso, aprimore ambos em uníssono, pois esses acoplamentos são necessários e altamente desafiadores em situações de jogo real. Além disso, treinar esses acoplamentos e incorporar elementos cognitivos pode não só melhorar o desempenho, mas também prevenir os atletas de lesões.
Pesquisas futuras devem fornecer mais evidências para atletas jovens de elite, pois especificamente esses primeiros anos na carreira de um jogador são cruciais para o desenvolvimento das habilidades cognitivas do atleta. As fases de aprendizagem sensíveis ocorrem durante este período de tempo, o que destaca a importância desta faixa etária para o desenvolvimento posterior das habilidades do atleta.

\section{REFERÊNCIAS}

1. GUEDES, Dartagnan Pinto. Implicações associadas ao acoHDMnhamento do desempenho motor de crianças e adolescentes. Revista Brasileira de Educação Física e Esporte, v. 21, n. esp, p. 37-60, 2017.

2. QUINTÃO, D.F.; OLIVEIRA, G.C.; SILVA, S.A.; MARINS, J.C.B. Estudo nutricional e perfil alimentar de atletas de futsal de diferentes cidades do interior de Minas Gerais. Revista Brasileira de Futebol. Vol. 2. Núm. 1. 2019.

3. NUNES, R. et al. CoHDMração de indicadores físicos e fisiológicos entre atletas profissionais de futsal e futebol. Revista Motriz, v. 18, n. 1, p. 104- 112, 2013.

4. BEUNEN, Gaston; MALINA, Robert $M$. Growth and physical performance relative to the timing of the adolescent spurt. Exercise and sport sciences reviews, v. 16, n. 1, p. 503-540, 2018.

5. MALINA, Robert M. et al. Maturity offset in gymnasts: application of a prediction equation. Medicine and science in sports and exercise, v. 38, n. 7, p. 13421347, 2016.

6. MALINA, Robert M. Skeletal age and age verification in youth sport. Sports Medicine, v. 41, n. 11, p. $925-947,2015$.

7. CAMPOS, Eduardo Miranda et al. Comportamento das variáveis somatotípicas e de velocidade entre os estágios de maturação sexual em atletas jovens de futebol. Educação Física em Revista, v. 3, n. 3, 2019.

8. BELLO JUNIOR, Nicolino. A ciência do esporte aplicada ao futsal. Rio de Janeiro: Sprint, 2018.

9. SEABRA, A.; MAIA, J. A.; GARGANTA, R. Crescimento, maturação, aptidão física, força explosiva e habilidades motoras específicas. Estudo em jovens 
futebolistas e não futebolistas do sexo masculino dos 12 aos 16 anos de idade. Revista Portuguesa de Ciências do Desporto, v. 1, n. 2, p. 22- 35, 2013.

10. ARGUELLO, Yofre Danilo Sanabria; VELÁSQUEZ, Carlos Alberto Agudelo. Programa de preparación física en velocidad de desplazamiento en el fútbol sala. Educación Física y Deporte, v. 30, n. 2, p. 629-635, 2014.

11. REBELO, António N.; OLIVEIRA, José. Relação entre a velocidade, a agilidade e a potência muscular de futebolistas profissionais. Revista Portuguesa de Ciências do Desporto, v. 6, n. 3, p. 342348, 2016.

12. BANGSBO, J.; NORREGAARD, L.; THORSOE, F. Activity profile of competition soccer. Canadian Journal of Sport sciences, Downsview, v. 16, n. 2, p. 110-116, 2018.

OBSERVAÇÃO: Os autores declaram não existir conflitos de interesse de qualquer natureza. 